\title{
Modeling and controlling the generator of a Thermoacoustic Stirling Engine
}

\author{
J.A. Brussen ${ }^{1}$ \\ ${ }^{1}$ Electrical Engineering, HAN University of Applied Sciences , Ruitenberglaan 26, \\ 6826 CC, Arnhem, The Netherlands. \\ Corresponding author's e-mail: johan.brussen@han.nl
}

Keywords: acoustic stirling engine, control, linear generator, efficiency

\section{Introduction}

The acoustic waves consist of alternating pressures and flows. The used generator is a linear generator that converts acoustic waves into electric power. The linear movement of the generator is limited; exceeding these limits might damage the generator.

The total system, consisting of the thermoacoustics engine, generator and load, is tuned to get acoustic waves with the desired frequency and amplitude.

A source with a temperature difference delivers the heat power to the system. This temperature difference changes in time. The power delivered depends on the input power and the loading of the system.

To avoid destruction of the generator, the movement of the generator has to be controlled. The load should also be controlled to maximize the output power.

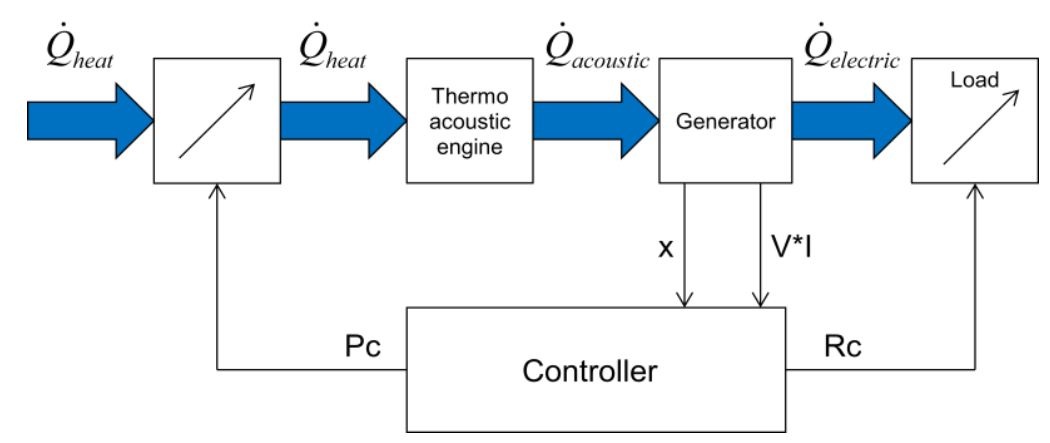

Figure 1: Block diagram of the system

\section{Controller design}

From the modeling it becomes clear a controller is needed to control the maximum electrical power delivered to the load. The maximum power is reached if the generator reaches its maximum excursion. The maximum power can be controlled by the value of the electrical load resistor. The control goal for this purpose can be stated as: Deliver maximum power to the electrical load resistor.

To deliver the maximum power to a given resistor the amplitude of the source has to be maximalized. At the start of the engine the amplitude is zero, to increase the amplitude the load resistance has to be higher than the calculated value. If the generator reaches its maximum excursion the load must be set to the calculated value. At this point the generated 
voltage is at its maximum so the maximum power is delivered to the resistor. The derived control goal becomes: Keep generator at maximum generated voltage.

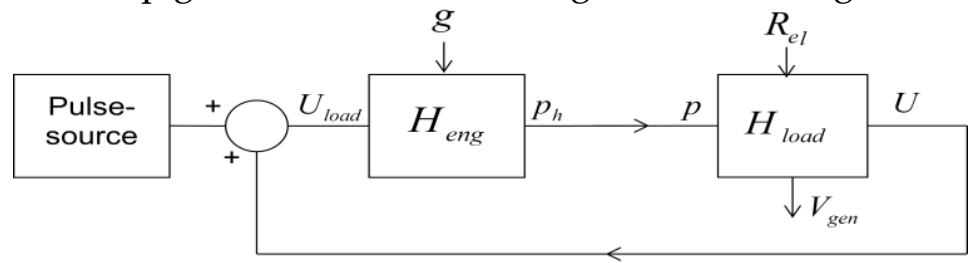

Figure 2: Process with controls

In Figure 2 the process is given. The feed forward loop make the system oscillate, the pulse source delivers the initial start-up energy. The temperature difference determines the gain of the engine $(g)$. The gain can change in time due to the change in the source of the wasted heat, $\mathrm{g}$ is considered to be a disturbance. $R_{l}$ is the input of the process and the $V_{g e n}$ the controlled variable. The process consists of a process with for $R_{>}>R_{0}$ the poles are in the right hand part of the pole plane, for $R_{l}<R_{0}$ the poles are be on the left site of the imaginary axis.. The controller keeps the poles on the imaginary axis. The system acts as an integrator. As the process is a non linear process, the process is linearized in an operating point. The operating point is given by the temperature difference. This difference dictates the $R_{l}$ for stationary operation. The integrator behavior of the process makes a P controller sufficient. The control scheme is given in Figure 3.

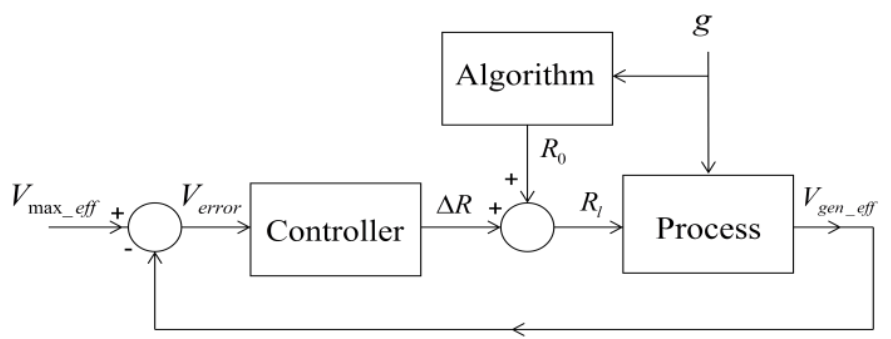

Figure 3: Control scheme

In this scheme, the output of the process is the rms value of the generated voltage, the $V_{\text {max_eff }}$ is calculated and used as set point of the system. The error voltage is fed to the controller. The output of the controller is added to the $R_{0} . R_{0}$ is the value of the load resistor at which steady state conditions are met. The algorithm calculates $R_{0}$.

If $R_{0}$ is calculated correctly, the controller drives the $V_{\text {gen_eff }}$ to the desired value, $V_{\text {error }}$ will be zero. There is no steady state error as the process introduces an integrating action.

\section{References}

[1] L. L. Beranek, Acoustics, Cambridge: Acoustical Society of America, 1993.

[2] K. Thorborg, A. D. Unruh and C. J. Struck, "An Improved Electrical Equivalent Circuit Model for Dynamic Moving Coil Transducers," in AES 122s Convention, Vienna, 2007.

[3] N. S. Nise, Control Systems Engineering, John Wiley and Sons Ltd, 2008.

[4] C. M. De Blok and R. F. M. Van Den Brink, "Full Characterization of Linear Acoustic Networks," J. Audio Eng. Soc., Vol. 40, No. 6, pp. 517-523, june 1992.

[5] S. Backhaus and G. W. Swift, "A thermoacoustic-Stirling heat engine: Detailed study," Acoustical Society of America, pp. 3148-3166, june 2000. 